\section{Fluorogenic probes for live-cell imaging of the cytoskeleton}

\author{
Gražvydas Lukinavičius $^{1,8}$, Luc Reymond ${ }^{1,2,8}$, \\ Elisa D'Este ${ }^{3}$, Anastasiya Masharina ${ }^{1}$, \\ Fabian Göttfert ${ }^{3}$, Haisen Ta ${ }^{3}$, Angelika Güther ${ }^{4}$, \\ Mathias Fournier ${ }^{5}$, Stefano Rizzo ${ }^{6}$, Herbert Waldmann ${ }^{6}$, \\ Claudia Blaukopf ${ }^{7}$, Christoph Sommer ${ }^{7}$, \\ Daniel W Gerlich ${ }^{7}$, Hans-Dieter Arndt ${ }^{4}$, Stefan W Hell ${ }^{3}$ \& \\ Kai Johnsson ${ }^{1,2}$
}

\begin{abstract}
We introduce far-red, fluorogenic probes that combine minimal cytotoxicity with excellent brightness and photostability for fluorescence imaging of actin and tubulin in living cells. Applied in stimulated emission depletion (STED) microscopy, they reveal the ninefold symmetry of the centrosome and the spatial organization of actin in the axon of cultured rat neurons with a resolution unprecedented for imaging cytoskeletal structures in living cells.
\end{abstract}

Live-cell microscopy of the cytoskeleton is important for studying processes such as cytokinesis, motility and organelle organization. The ideal probe for cytoskeleton imaging is highly fluorogenic and nontoxic, has far-red emission and excitation wavelengths, and labels with high specificity in living cells. Up to now, small molecules that fulfill this wish list have not been described. Probes for the two major components of the cytoskeleton, tubulin and actin, have been introduced by linking fluorophores to taxanes and phalloidines that bind to microtubules and F-actin filaments, respectively ${ }^{1-3}$. However, current paclitaxel (Taxol) derivatives do not show increased fluorescence upon target binding (i.e., they are not fluorogenic $)^{4}$, and phalloidin derivatives are not cell perme$\mathrm{able}^{2}$, thereby resulting in limited applicability in both cases.

Our design of probes for live-cell imaging of the cytoskeleton is based on recently reported silicon-rhodamine ( $\mathrm{SiR}$ ) derivatives $^{5}$. SiR derivatives exist in equilibrium between a nonfluorescent spirolactone (OFF state) and a fluorescent zwitterion (ON state). Aggregation of $\mathrm{SiR}$ derivatives or their unspecific binding to hydrophobic surfaces favors the OFF state, whereas their interaction with polar protein surfaces switches the fluorophores into the ON state. We conjugated $\mathrm{SiR}$ to the ligands docetaxel ${ }^{6}$ and desbromo-desmethyl-jasplakinolide ${ }^{7}$, which bind to microtubules and to F-actin, respectively (Supplementary Fig. 1a). By systematically varying the nature of the hydrophobic linker between the fluorophore and the targeting ligand (Supplementary Notes 1 and 2), we identified the fluorogenic probes SiR-tubulin and SiR-actin (Fig. 1a). SiR-tubulin increased its fluorescence intensity more than tenfold when binding to microtubules in vitro, whereas SiR-actin displayed an increase in fluorescence of more than 100-fold (Fig. 1b and Supplementary Figs. $\mathbf{1 b}$ and $\mathbf{2 c , d}$ ). We measured a similar fluorescence increase when surfactant was added (Supplementary Figs. 1b and 2), which is in agreement with a mechanism of ON-OFF switching by aggregation ${ }^{5}$.

SiR-tubulin and SiR-actin were suitable for live-cell imaging, as demonstrated for human primary dermal fibroblasts (Fig. 1c). These cells are difficult to transfect, making imaging of their cytoskeleton with genetically encoded probes challenging. Specific and comprehensive labeling of microtubules and actin filaments by $\mathrm{SiR}$ probes at concentrations of $2 \mu \mathrm{M}$ was also validated in HeLa cells (Supplementary Fig. 3). Additionally, in five out of ten cell lines from five different organisms tested, SiR-tubulin and SiR-actin permitted efficient staining of the cytoskeleton (Supplementary Table 1). In four out of the five cell lines that showed low labeling efficiency, staining was substantially improved by addition of verapamil, a broad-spectrum efflux-pump inhibitor ${ }^{8}$, a result indicating that labeling efficiency with SiR probes depends on the expression level of efflux pumps. Furthermore, the fluorogenicity of the probes permitted imaging without removal of excess probe through washing (Supplementary Figs. 4 and 5).

In a further application, we stained the actin cytoskeleton of intact erythrocytes with $5 \mu \mathrm{M}$ SiR-actin in whole-blood samples (Supplementary Fig. 6). Imaging of the actin cytoskeleton in these cells benefited from three key properties of SiR-probes. First, their far-red excitation and emission wavelengths avoid interference with the absorbance spectrum of hemoglobin. Second, SiR-based probes can be used directly on samples that are difficult to transfect, such as erythrocytes. Third, the fluorogenic character of the probes permits their use without any washing steps.

Applicability of our $\mathrm{SiR}$ probes to the study of cytoskeletal dynamics requires low toxicity. Docetaxel, jasplakinolide and their derivatives have been shown to be cytotoxic and antineoplastic $^{9,10}$, but derivatization of docetaxel at the $3^{\prime} N$ position with a fluorophore reduces cell toxicity ${ }^{6}$. Likewise, synthetic desbromodesmethyl-jasplakinolide derivatives conjugated to fluorophores

\footnotetext{
${ }^{1}$ Institute of Chemical Sciences and Engineering, École Polytechnique Fédérale de Lausanne, Lausanne, Switzerland. ${ }^{2}$ National Centre of Competence of Research in Chemical Biology, Lausanne, Switzerland. ${ }^{3}$ Department of NanoBiophotonics, Max Planck Institute for Biophysical Chemistry, Göttingen, Germany. ${ }^{4}$ Institute of Organic Chemistry and Macromolecular Chemistry, Friedrich Schiller University, Jena, Germany. ${ }^{5}$ Bioimaging and Optics Platform, École Polytechnique Fédérale de Lausanne, Lausanne, Switzerland. ${ }^{6}$ Max Planck Institute of Molecular Physiology, Dortmund, Germany. ${ }^{7}$ Institute of Molecular Biotechnology of the Austrian Academy of Sciences, Vienna, Austria. ${ }^{8}$ These authors contributed equally to this work. Correspondence should be addressed to K.J. (kai.johnsson@epfl.ch), H.D.-A. (hd.arndt@uni-jena.de) or S.W.H. (shell@gwdg.de).
} 
Figure 1 SiR-tubulin and SiR-actin.

(a) Structures of SiR-tubulin and SiR-actin.

(b) Excitation and emission spectra of SiRtubulin and $\mathrm{SiR}$-actin probes in the presence of polymerized tubulin (monomer concentration, $2 \mathrm{mg} / \mathrm{ml}$ ) or F-actin (monomer concentration, $0.4 \mathrm{mg} / \mathrm{ml}$ ) as well as in the presence of bovine serum albumin (BSA; 2 or $0.4 \mathrm{mg} / \mathrm{ml}$, respectively). (c) Structured illumination microscopy (SIM) images of human fibroblasts stained with SiR-tubulin (top) or SiR-actin (bottom). Cells were incubated with $2 \mu \mathrm{M}$ probes in growth medium for $1 \mathrm{~h}$ and washed once before imaging. Scale bars, $5 \mu \mathrm{m}$.

only minimally impair actin dynamics in living cells ${ }^{7}$. In agreement with these data, SiR-tubulin and SiR-actin probes displayed attenuated influence on actin and tubulin polymerization kinetics in in vitro assays (Supplementary Fig. 7). At the concentrations used for long-term imaging, neither SiR-tubulin nor SiR-actin exhibited substantial cytotoxicity toward primary human fibroblasts over a period of 24 h (Supplementary Note 3 and Supplementary Fig. 8). SiR probes showed no detectable effect at the imaging concentrations on proliferating HeLa cells expressing a chromatin marker (histone $2 \mathrm{~B}$ (H2B)-mRFP) and a plasma-membrane marker (MyrPalm-mEGFP) $)^{11}$ (Supplementary Fig. 9 and Supplementary Videos 1-3).

Next we tested whether SiR-probes interfere with the formation of the mitotic cytoskeleton. We observed normal metaphase and anaphase spindle morphology with $100 \mathrm{nM}$ SiR-tubulin (Supplementary Fig. 3a). Furthermore, up to 100 nM SiR-tubulin had little effect on mitotic duration from prometaphase until anaphase (Supplementary Fig. 9), a result validating assembly of functional mitotic spindles. In contrast, treatment with the microtubule-stabilizing compound Taxol substantially prolonged mitosis even at $1 \mathrm{nM}$ (Supplementary Fig. 9 and Supplementary Videos 2 and 4). These data validate SiR-tubulin as a marker for imaging functional mitotic spindles.

\section{b}
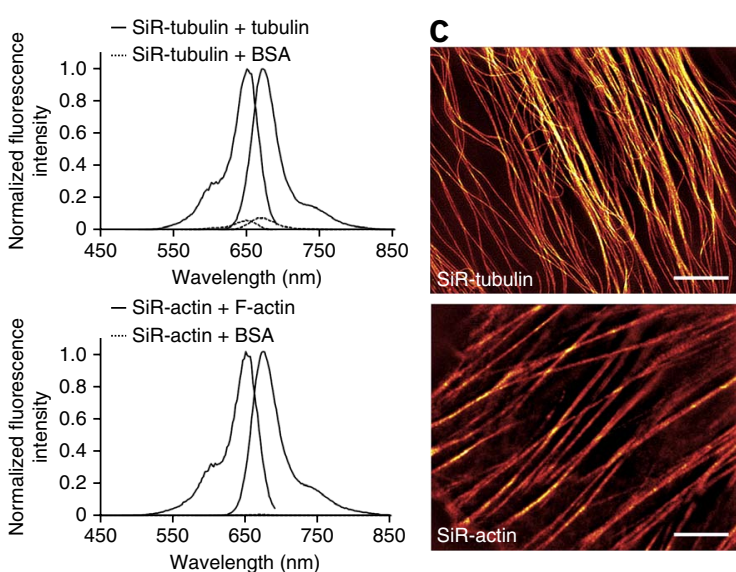

SiR-actin had little effect on mitotic duration at concentrations tested up to $3.2 \mu \mathrm{M}$, yet SiR-actin concentrations higher than $100 \mathrm{nM}$ reduced cell proliferation rates (Supplementary Fig. 9 and Supplementary Videos 3 and 5). Normal appearance of the cleavage furrow was observed in $99 \%$ of the cells $(n=644)$ in the presence of $100 \mathrm{nM}$ SiR-actin (Supplementary Fig. 3b), whereas jasplakinolide applied at these concentrations prevented cleavage furrow ingression in all cells $(n=29$; Supplementary Fig. 9). Furthermore, the fraction of multinucleated cells, which indicates failed cytokinesis, increased only slightly after $23 \mathrm{~h}$ of treatment with $100 \mathrm{nM} \mathrm{SiR-actin}(3.5 \% \pm 0.3 \%$ in SiR-actin com pared to $1.8 \% \pm 0.4 \%$ in untreated controls; $n \geq 1,000$ cells; \pm s.d. given throughout). These data demonstrate that SiR-actin can be used to visualize the dynamics of functional actin networks.

The high light intensities used in many microscopy techniques can result in phototoxicity ${ }^{12}$. We did not observe increased phototoxicity for either SiR probe at the conditions used for regular long-term time-lapse imaging: only at a tenfold higher light dose and at high probe concentrations did we detect increased phototoxicity (Supplementary Fig. 9f,g). The low phototoxicity
Figure 2 | Live-cell STED microscopy with SiR-tubulin and SiR-actin. (a) Representative STED image of centrosomal microtubules after Richardson-Lucy deconvolution; the observed ring is a projection of the centriole along its longitudinal axis. The diameter of the centriole and the polar angle between neighboring maxima of fluorescence intensity along the periphery of the centriole are indicated (see Supplementary Fig. 11). Cells were incubated with $2 \mu \mathrm{M}$ SiR-tubulin in growth medium for $1 \mathrm{~h}$ and washed once before imaging. Scale bar, $200 \mathrm{~nm}$. (b) Measured centriolar diameter of human $(n=18, N=17)$ and mouse $(n=11, N=11)$ centrosomes. Mean \pm s.d. is shown for $\mathbf{b}, \mathbf{c}, \mathbf{f}$. $n$, number of measured centrosomes; $N$, number of measured cells. (c) Measured polar angles between neighboring maxima of fluorescence intensity along the periphery of human $(n=53, N=7)$
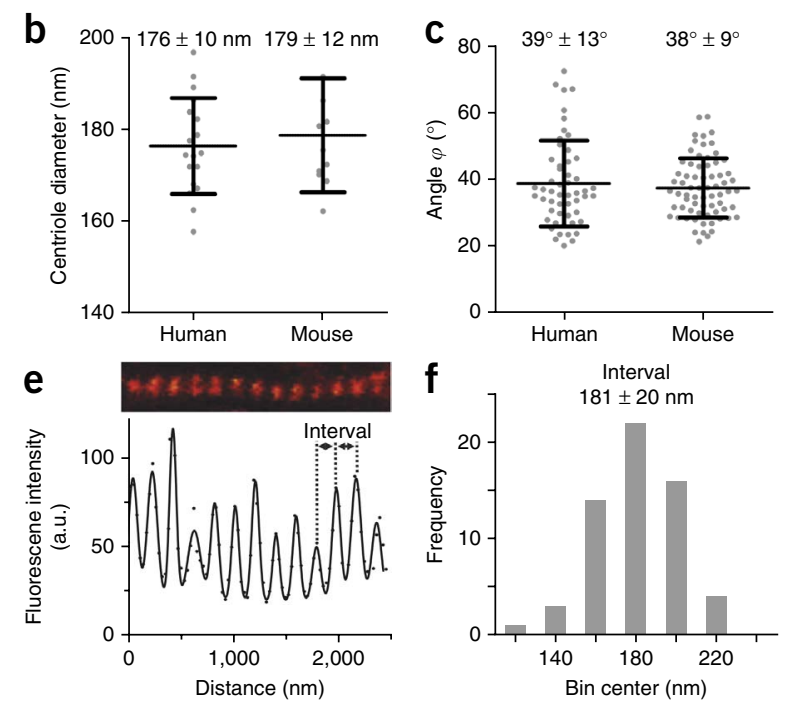
( $n=69, N=9)$ centriole, $n$, number of measured angles: $N$, number of centrosomes measured. (d) STED image (raw data) showing axons of rat primary hippocampal neurons stained with SiR-actin at $16 \mathrm{~d}$ in vitro. Actin rings are visible as stripes in several axons. Cells were incubated with $2 \mu \mathrm{M}$ SiR-actin in growth medium for $1 \mathrm{~h}$ and washed once before imaging. Scale bar, $1 \mu \mathrm{m}$. (e) Measured intensity signal profile of SiR-actin stripes fitted to multiple Gaussian distributions, and estimation of the distance (interval) between neighboring peaks. a.u., arbitrary units. (f) Distribution histogram of measured interpeak distances $(n=60, N=7)$. $n$, number of measured distances; $N$, number of measured axons. 
of the probes makes them attractive for live-cell super-resolution microscopy. Using structured illumination microscopy (SIM), we imaged human fibroblasts stained with SiR-tubulin and SiR-actin (Fig. 1c). The obtained microtubule diameter of $126 \pm 18 \mathrm{~nm}$ corresponds to the maximal resolution achievable with SIM ${ }^{13}$ (Supplementary Fig. 10a-c). We also imaged microtubule and actin remodeling in human fibroblasts by SIM time-lapse movies (Supplementary Videos 6 and 7).

The high photostability of SiR-actin and SiR-tubulin renders them particularly suitable for imaging by STED microscopy ${ }^{14,15}$. Staining of living human fibroblasts with SiR-tubulin and subsequent STED imaging highlighted peripheral microtubules and the microtubules of the centrosome (Fig. 2a and Supplementary Fig. 10d,g). The apparent microtubule diameter was $39 \pm 10 \mathrm{~nm}$ (Supplementary Fig. 10d-f), which, to our knowledge, indicates the highest resolution achieved so far at imaging microtubules in living cells, essentially doubling the resolution attained by $\mathrm{SiR}$ labeling of a SNAP-tag fusion of the microtubule-binding protein Cep41 (ref. 5). The higher resolution achieved with SiR-tubulin underscores that the use of small-molecule probes that directly target the structure of interest enhances the resolution substantially. Furthermore, we recorded time-lapse STED image series, thereby demonstrating that SiR-tubulin can be used to monitor microtubule dynamics via STED (Supplementary Video 8). The structure of the centrosome is sensitive to environmental conditions, and its characterization in living cells thus avoids potential fixation artifacts ${ }^{16}$. The centrosome is built around the centriole, a cylindrical structure composed of nine triplets of microtubules ${ }^{17}$. Imaging the centrosome in human fibroblasts or in mouse IA32 cells revealed rings of $176 \pm 10 \mathrm{~nm}$ and $179 \pm 12 \mathrm{~nm}$ in diameter, respectively (Fig. 2b and Supplementary Figs. 10h,i and 11). The diameter of these rings is in agreement with the previously reported values for the diameter of the centriole obtained by electron microscopy ${ }^{16}$. Furthermore, the STED data showed a pronounced modulation in brightness along the perimeter of the ring (Fig. 2a and Supplementary Fig. 11). The measured polar angle $\varphi$ between two neighboring maxima equaled $39^{\circ} \pm 13^{\circ}$ and $38^{\circ} \pm$ $9^{\circ}$ for human and mouse centrosomes, respectively (Fig. 2c). This angle is consistent with the ninefold symmetry of the centriole and is close to the previously measured $42^{\circ} \pm 11^{\circ}$ obtained by electron microscopy ${ }^{17}$. To our knowledge, this is the first visualization of the centriole's ninefold symmetry in living cells.

Recent stochastic optical reconstruction microscopy (STORM) on the organization of actin in fixed neurons revealed that actin formed ring-shaped structures at the rim of axons in neuronal cultures. These structures are evenly spaced along axonal shafts with a periodicity of $\sim 180-190 \mathrm{~nm}$ (ref. 18). To investigate the actin spatial arrangement under live-cell conditions, we labeled primary rat hippocampal neurons with $2 \mu \mathrm{M}$ SiR-actin. Subsequent STED imaging revealed periodic structures along the axons (Fig. 2d,e and Supplementary Fig. 12) with a periodicity of $181 \pm 20 \mathrm{~nm}$ (Fig. 2f) in living cells, which is in excellent agreement with reported data from fixed phalloidin-stained neurons ${ }^{18}$. We failed to visualize these periodic actin structures with the popular actin marker Lifeact ${ }^{19}$ (Supplementary Fig. 13), underscoring the utility of SiR-actin for live-cell fluorescence super-resolution STED microscopy.
In conclusion, $\mathrm{SiR}$-actin and $\mathrm{SiR}$-tubulin are fluorogenic and farred probes that display minimal toxicity in live-cell experiments. In combination with super-resolution fluorescence microscopy, they can be used to image cytoskeletal structures in living cells and tissues at an unprecedented level of detail. For all these reasons, SiR-actin and SiR-tubulin should be beneficial for bioimaging.

\section{METHODS}

Methods and any associated references are available in the online version of the paper.

Note: Any Supplementary Information and Source Data files are available in the online version of the paper.

\section{ACKNOWLEDGMENTS}

The authors are grateful to J.E. Bear (University of North Carolina at Chapel Hill), P. Gönczy (École Polytechnique Fédérale de Lausanne (EPFL)), R. Jahn (University of Göttingen), D. Abankwa (Åbo Akademi University), U. Ruegg (University of Geneva) and A. Seitz (EPFL) for sharing reagents and for technical assistance. K.J. acknowledges support from the Swiss National Science Foundation and the National Centre of Competence of Research (NCCR) Chemical Biology. Support from the Körber foundation was received through the European Science Prize to S.W.H. D.W.G. acknowledges support from the European Community's Seventh Framework Programme FP7/2007-2013 under grant agreements nos. 241548 (MitoSys) and 258068 (Systems Microscopy) and from an European Research Council (ERC) Starting Grant (agreement no. 281198).

\section{AUTHOR CONTRIBUTIONS}

G.L., L.R. and K.J. devised this study. All authors except S.R. and A.G. contributed to manuscript writing. G.L., E.D., A.M., C.B., C.S. and D.W.G. characterized the probes. L.R., A.G. and S.R. performed synthesis of probes, supervised by K.J., H.W. and H.-D.A.; G.L., E.D., F.G. and H.T. performed STED microscopy, guided by S.W.H.; G.L. and M.F. performed SIM microscopy.

\section{COMPETING FINANCIAL INTERESTS}

The authors declare competing financial interests: details are available in the online version of the paper.

Reprints and permissions information is available online at http://www.nature. com/reprints/index.html.

1. Barasoain, I., Díaz, J.F. \& Andreu, J.M. Methods Cell Biol. 95, 353-372 (2010).

2. Huang, Z.J., Haugland, R.P. \& You, W.M. Anal. Biochem. 200, 199-204 (1992).

3. Wulf, E., Deboben, A., Bautz, F.A., Faulstich, H. \& Wieland, T. Proc. Natl. Acad. Sci. USA 76, 4498-4502 (1979).

4. Díaz, J.F., Barasoain, I., Souto, A.A., Amat-Guerri, F. \& Andreu, J.M. J. Biol. Chem. 280, 3928-3937 (2005).

5. Lukinavičius, G. et al. Nat. Chem. 5, 132-139 (2013).

6. Dubois, J. et al. Bioorg. Med. Chem. 3, 1357-1368 (1995).

7. Milroy, L.G. et al. J. Am. Chem. Soc. 134, 8480-8486 (2012).

8. Bellamy, W.T. Annu. Rev. Pharmacol. Toxicol. 36, 161-183 (1996).

9. Cortes, J.E. \& Pazdur, R. J. Clin. Oncol. 13, 2643-2655 (1995).

10. Bubb, M.R., Senderowicz, A.M., Sausville, E.A., Duncan, K.L. \& Korn, E.D. J. Biol. Chem. 269, 14869-14871 (1994).

11. Steigemann, P. et al. Cell 136, 473-484 (2009).

12. Schneckenburger, H. et al. J. Microsc. 245, 311-318 (2012).

13. Gustafsson, M.G. J. Microsc. 198, 82-87 (2000).

14. Klar, T.A., Jakobs, S., Dyba, M., Egner, A. \& Hell, S.W. Proc. Natl. Acad. Sci. USA 97, 8206-8210 (2000).

15. Hell, S.W. \& Wichmann, J. Opt. Lett. 19, 780-782 (1994).

16. Paintrand, M., Moudjou, M., Delacroix, H. \& Bornens, M. J. Struct. Biol. 108, 107-128 (1992).

17. Kitagawa, D. et al. Cell 144, 364-375 (2011).

18. Xu, K., Zhong, G. \& Zhuang, X. Science 339, 452-456 (2013).

19. Riedl, J. et al. Nat. Methods 5, 605-607 (2008). 


\section{ONLINE METHODS}

In vitro tubulin polymerization assay. Measurement of the enhancement of the rates of tubulin polymerization by the probes was performed using a commercial tubulin polymerization fluorescence assay kit available from Cytoskeleton, Inc. (cat. BK011P). It is based on DAPI (4',6-diamidino-2-phenylindole) fluorescence change upon tubulin polymerization ${ }^{20}$. All measurements were carried out according to manufacturer recommendations using standard conditions protocol. Samples were measured in a halfarea 96-well plate (Greiner Bio-One, cat. 675076) on an Infinite M1000 spectrofluorometer (Tecan). Fluorescence emission was detected at $430 \mathrm{~nm}$ while exciting at $350 \mathrm{~nm}$. The excitation and emission bandwidth for all measurements was set to 20 and $10 \mathrm{~nm}$, respectively. During acquisition the temperature was set to $37^{\circ} \mathrm{C}$. All samples were prepared in duplicates, and fluorescence was measured over $90 \mathrm{~min}$ (one measurement each minute) until a stable signal was achieved. The lowest value of each data set was normalized to 1 . Obtained polymerization curves were analyzed using GraphPad Prism 6.0 version and fitted to a model describing plateau followed by one-phase exponential association:

$$
y=y_{0}+\left(\text { plateau }-y_{0}\right)\left(1-e^{-K\left(x-x_{0}\right)}\right)
$$

considering that $x_{0}$ is the time at which tubulin polymerization begins, $y_{0}$ is the average $y$ value up to time $x_{0}$, 'plateau' is the $y$ value at infinite times and $K$ is the rate constant. Obtained results are summarized (Supplementary Fig. 7 and Supplementary Table 2).

In vitro actin polymerization and depolymerization assay. The influence of the probes on the rate of actin polymerization and depolymerization was assessed using a commercial actin polymerization fluorescence assay kit available from Cytoskeleton (cat. BK003). It is based on pyrene-labeled actin fluorescence change upon polymerization or depolymerization ${ }^{21}$. Measurements were performed in 96-well plates (Greiner Bio-One, cat. 655900) on an Infinite M1000 spectrofluorometer. Fluorescence emission was detected at $420 \mathrm{~nm}$ while exciting at $350 \mathrm{~nm}$. The excitation and emission bandwidth for all measurements was set to $20 \mathrm{~nm}$, and the temperature was set to $37^{\circ} \mathrm{C}$. All samples were prepared in triplicates, and fluorescence was measured over $4 \mathrm{~h}$ (one measurement every $2 \mathrm{~min}$ ). First, G-actin (monomeric actin) was prepared according to manufacturer recommendation by dissolving lyophilized rabbit pyrene-labeled muscle actin in G-buffer (5 mM Tris- $\mathrm{HCl}$ ( $\mathrm{pH} 8.0$ ), $0.2 \mathrm{mM} \mathrm{CaCl}_{2}$ and $0.2 \mathrm{mM} \mathrm{ATP}$ ) to a final concentration of $0.4 \mathrm{mg} / \mathrm{ml}$. Subsequently, G-actin was mixed with probes (final concentration: $5 \mu \mathrm{M}$ ), and pyrene fluorescence was measured until a stable signal was achieved. G-actin polymerization was induced by the addition of actin polymerization buffer (for final buffer composition of $50 \mathrm{mM} \mathrm{KCl}, 2 \mathrm{mM}$ $\mathrm{MgCl}_{2}, 5 \mathrm{mM}$ guanidine carbonate and $1 \mathrm{mM} \mathrm{ATP}$ ), and pyrene fluorescence was measured until completion of polymerization $(\sim 1 \mathrm{~h})$. Depolymerization of F-actin (filamentous actin) was induced by a fivefold dilution of the samples with G-buffer, and pyrene fluorescence was measured for $3 \mathrm{~h}$ until fluorescent signals reached a plateau. For polymerization curves the lowest value of each data set was normalized to 1 , and for depolymerization curves the highest value of each data set was normalized to 1 . Obtained polymerization and depolymerization curves were fitted using GraphPad Prism 6.0. Polymerization curves were fitted to the equation (1) describing 'plateau followed by one-phase exponential association'. Depolymerization curves were fitted to the model describing two-phase decay:

$$
\begin{aligned}
y= & \text { plateau }+\left(y_{0}-\text { plateau }\right) 0.01 \alpha e^{-K_{\text {fast }} x}+ \\
& \left(y_{0}-\text { plateau }\right)(100-\alpha) 0.01 e^{-K_{\text {slow }} x}
\end{aligned}
$$

considering that $y_{0}$ is the $y$ value when $x$ (time) is 0 , plateau is the $y$ value at infinite times, $K_{\text {fast }}$ and $K_{\text {slow }}$ are the two rate constants and $\alpha$ is the fraction of the span (from $y_{0}$ to plateau) accounted for by the faster of the two components. Obtained results are summarized (Supplementary Fig. 7 and Supplementary Table 2).

Estimation of fluorescence increase upon target binding. Substrate from a $250 \mu \mathrm{M}$ DMSO (Applichem, cat. A3672) stock solution was directly added to the target protein $(0.4 \mathrm{mg} / \mathrm{ml}$ $\mathrm{G}$-actin or $2 \mathrm{mg} / \mathrm{ml}$ monomeric tubulin), to $0.2 \% \mathrm{SDS}$ (Applichem $\mathrm{GmbH}$, cat. A1502) or to a bovine serum albumin (BSA) (Sigma, cat. B4287) solution $(0.4 \mathrm{mg} / \mathrm{ml}$ or $2 \mathrm{mg} / \mathrm{ml})$ (1:83 dilution). In the case of tubulin probes, buffer containing $80 \mathrm{mM}$ piperazine$N, N^{\prime}$-bis(2-ethanesulfonic acid) sequisodium salt (PIPES) (Applichem, cat. A3495), $2 \mathrm{mM} \mathrm{MgCl}_{2}, 0.5 \mathrm{mM}$ ethylene glycol-bis ( $\beta$-aminoethyl ether) $N, N, N^{\prime}, N^{\prime}$-tetra-acetic acid (EGTA, pH 6.9) (Applichem, cat. A0878), 1 mM GTP (Cytoskeleton, cat. BST06) and 15\% glycerol (Cytoskeleton, cat. BST05) was used. In the case of actin probes, buffer containing $5 \mathrm{mM}$ Tris- $\mathrm{HCl}$ ( $\mathrm{pH}$ 8.0), $0.2 \mathrm{mM} \mathrm{CaCl}_{2}$ and $0.2 \mathrm{mM}$ ATP was used. This buffer was supplemented with $50 \mathrm{mM} \mathrm{KCl}, 2 \mathrm{mM} \mathrm{MgCl}$, $5 \mathrm{mM}$ guanidine carbonate and $1 \mathrm{mM}$ ATP to obtain F-actin. Both buffers are components of the actin polymerization fluorescence assay kit (Cytoskeleton, cat. BK003). The samples prepared in 1.5-ml tubes (Eppendorf) were incubated for $2-3 \mathrm{~h}$ at $37^{\circ} \mathrm{C}$, and fluorescence was measured in a half-area 96-well plate (Greiner Bio-One, cat. 675076) on an Infinite M1000 spectrofluorometer. Fluorescence emission was recorded from $580 \mathrm{~nm}$ to $850 \mathrm{~nm}$ while exciting at $550 \mathrm{~nm}$. Fluorescence excitation was recorded by measuring the emission at $720 \mathrm{~nm}$ while exciting from $450 \mathrm{~nm}$ to $690 \mathrm{~nm}$. The excitation and emission bandwidth for all measurements was set to $10 \mathrm{~nm}$. All samples were prepared in triplicates. Ratios $F_{(+\mathrm{SDS})} /$ $F_{(+\mathrm{BSA})}$ or $F_{(+ \text {enzyme })} / F_{(+\mathrm{BSA})}$ of fluorescence signals at $672 \mathrm{~nm}$ were calculated. Obtained results are summarized (Supplementary Figs. $\mathbf{1 b}$ and $\mathbf{2}$ and Supplementary Table 2).

LogD calculation. $\log \mathrm{D}$ at $\mathrm{pH} 7.5$ was calculated using MarvinSketch 6.0.5 (ref. 22, http://www.chemaxon.com/). Chemical structures of the probes that were used for the calculation are displayed in Supplementary Figure 1. Obtained results are summarized (Supplementary Fig. 1b and Supplementary Table 3)

Estimation of quantum yield of the probes. Absorbance and fluorescence emission (excitation at $550 \mathrm{~nm}$ ) spectra of an approximately $15 \mu \mathrm{M}$ solution of probes 1-9 (Supplementary Fig. 1a) in TBS (50 mM Tris-HCl pH 7.4 (Sigma, cat. T7693), $150 \mathrm{mM} \mathrm{NaCl}$ (Fluka, cat. 71383)) + $1 \mathrm{mg} / \mathrm{ml} \mathrm{BSA}$ and with or without $0.2 \%$ SDS were recorded on an Infinite M200Pro spectrofluorometer. The spectra were plotted and integrated using the software Mathematica (Wolfram). Absorbance spectra were 
integrated from 550 to $720 \mathrm{~nm}$, and fluorescence spectra were integrated from 580 to $800 \mathrm{~nm}$. The reported quantum yield of SiR-COOH in PBS (Lonza, cat. BE17-516F) of 0.4 was used as reference ${ }^{5}$. Quantum yields (QY) were calculated using the following equation:

$$
\mathrm{QY}=0.4 \frac{\text { Int }_{\text {Em,probe }}{ }^{\text {Int }_{\mathrm{Abs}, \mathrm{ref}}}}{\text { Int }_{\mathrm{Abs}, \text { probe }}{ }^{\text {Int }_{\mathrm{Em}, \mathrm{ref}}}}
$$

where Int $_{E m \text {,probe }}$ is the integral of the emission spectrum of the probe; Int $_{\mathrm{Abs} \text {,probe }}$ is the integral of the absorption spectrum of the probe; Int $_{\mathrm{Em} \text {,ref }}$ is the integral of the reference emission spectrum; and Int $_{\mathrm{Abs} \text {,ref }}$ is the integral of the reference absorption spectrum. Obtained results are summarized (Supplementary Table 3).

Preparation and maintenance of cells. Human primary dermal fibroblasts were cultured in high-glucose DMEM (Life Technologies, cat. 31053-028) supplemented with GlutaMAX-1 (Life Technologies, cat. 35050-038) 10\% FBS (FBS) (Life Technologies, cat. 10270-106) in a humidified 5\% $\mathrm{CO}_{2}$ incubator at $37{ }^{\circ} \mathrm{C}$. Cells were split every $3-4 \mathrm{~d}$ or at confluence. Cells were seeded in glass-bottom 12-well or 24-well plates (MatTek, cat. P12GC-1.0-14-F or P24G-1.5-13-F) or glass-bottom 35-mm dishes (MatTek, cat. P35GC-1.5-10-C) $1 \mathrm{~d}$ before imaging.

Transduction with CellLight Tubulin-GFP (Life Technologies, cat. C10613) and CellLight Actin-RFP reagents (Life Technologies, cat. C10583) was performed according to manufacturer recommendations. $5 \mu \mathrm{l}$ per well of CellLight reagent was added to $0.5 \mathrm{ml}$ of complete DMEM medium in a 24 -well plate; $10 \mu \mathrm{l}$ per well was added to $1 \mathrm{ml}$ of complete DMEM medium in a 12 -well plate. Afterwards, cells were grown for $24 \mathrm{~h}$ in a humidified $5 \% \mathrm{CO}_{2}$ incubator at $37^{\circ} \mathrm{C}$. Staining of GFP-tubulin or RFP-actin expressing cells with SiR-based probes was performed as described below.

Stably expressing HeLa cell lines were derived from the HeLa 'Kyoto' cell line obtained from S. Narumiya (Kyoto University, Japan). Cell lines stably coexpressing H2B-mRFP and MyrPalmmEGFP, or H2B-mRFP and mEGFP- $\alpha$-tubulin, or H2B-mCherry and actin-EGFP described in ref. 11 were used. HeLa Kyoto cells were cultured in DMEM supplemented with 10\% (v/v) FBS, 1\% (v/v) penicillin-streptomycin (pen/strep; Sigma), $500 \mu \mathrm{g} / \mathrm{ml} \mathrm{G418}$ and $0.5 \mu \mathrm{g} / \mathrm{ml}$ puromycin. For live-cell microscopy, cells were grown either in 96-well plastic-bottom plates ( $\mu$ clear; Greiner BioOne) or on LabTek II chambered coverslips (Thermo Scientific). Live-cell imaging was performed in DMEM containing $10 \%(\mathrm{v} / \mathrm{v})$ FBS and $1 \%(\mathrm{v} / \mathrm{v})$ pen/strep, but without phenol red and riboflavin to reduce autofluorescence.

Cultures of hippocampal neurons were prepared from Wistar rats of mixed sex at postnatal day $\mathrm{P} 0-\mathrm{P} 1$ in accordance with the regulations of the German Animal Welfare Act and under the approval of the local veterinary service. Cells were plated on $100 \mu \mathrm{g} / \mathrm{ml}$ polyornithine (Sigma-Aldrich, cat. P3655) and $1 \mu \mathrm{g} / \mathrm{ml}$ laminin (BD Bioscience, cat. 354232)-coated coverslips. Neuronal cultures were maintained in Neurobasal medium (Gibco, cat. 21103049) supplemented with 2\% B27 serum-free supplement (Gibco, cat. 17504044), 2 mM L-glutamine (Gibco, cat. 25030) and pen/strep (100 units/ml and $100 \mu \mathrm{g} / \mathrm{ml}$, respectively, BiochromAG, cat. A2213). The day after plating, $5 \mu \mathrm{M}$ cytosine $\beta$-D-arabinofuranoside (Sigma, cat. C1768) was added to the cultures. Medium was replaced once per week.
Cell sources are listed in Supplementary Table 1. All the cell lines were mycoplasma negative (periodically checked). Primary hippocampal neurons were not checked for mycoplasma infection.

Preparation of erythrocytes. Mammalian and chicken erythrocytes shape is maintained by the membrane skeleton, which can be stained with the SiR-actin probe ${ }^{23,24}$. Heparin-treated human (InnovativeResearch, cat. IPLA-WB1), mouse (InnovativeResearch, cat. IR1-993N) and chicken (InnovativeResearch, cat. IR1-080N) whole blood was diluted 1:500 with RBC buffer (10 mM HEPES, $154 \mathrm{mM} \mathrm{NaCl}, 0.1 \%$ glucose and 1\% BSA) in Eppendorf tubes. SiR-actin from a DMSO stock was added to the final concentration of $5 \mu \mathrm{M}$ and incubated for $30 \mathrm{~min}$ at room temperature (RT). The suspension was transferred to a 24-well glass-bottom dish and imaged directly without washing. For confocal imaging erythrocytes were stained with $1 \mu \mathrm{M}$ SiR-actin and $1 \mu \mathrm{M}$ Bodipy FL C5-ceramide complexed to BSA (Life Technologies, cat. B22650) for $60 \mathrm{~min}$ at RT. The mix was transferred to a 35-mm glass-bottom dish and imaged. Stained erythrocytes were imaged using fluorescence microscopy, and results are summarized (Supplementary Fig. 6).

Staining of living and fixed cell lines. Live-cell staining with tubulin or actin probes was achieved by simply adding the probes from a $1 \mathrm{mM}$ DMSO stock solution to the complete growth medium to obtain the desired final concentration (usually 1-3 $\mu \mathrm{M}$ ) and incubating for $1 \mathrm{~h}$ in a humidified $5 \% \mathrm{CO}_{2}$ incubator at $37^{\circ} \mathrm{C}$. If required, Hoechst 33342 (Life Technologies, cat. H1399) was added together with probes at the final concentration of $1 \mu \mathrm{g} / \mathrm{ml}$.

Methanol fixation was performed as follows: growth medium was removed from cells, cells were incubated for 3-10 min in $-20{ }^{\circ} \mathrm{C}$ cold methanol containing $5 \mathrm{mM}$ EGTA, washed once with PBS and blocked for $30 \mathrm{~min}$ with $1 \%$ BSA in PBS. Subsequently, samples were stained for 60 min with $2 \mu \mathrm{M}$ probes and Hoechst 33342 $(1 \mu \mathrm{g} / \mathrm{ml})$ dissolved in PBS containing $1 \%$ BSA.

Paraformaldehyde fixation was performed as follows: growth medium was removed from cells, and then cells were incubated for $10 \mathrm{~min}$ in PBS containing 4\% formaldehyde, washed once with PBS and blocked for $30 \mathrm{~min}$ in 1\% BSA in PBS. Subsequently, samples were stained for $60 \mathrm{~min}$ with $2 \mu \mathrm{M}$ probes and Hoechst $33342(1 \mu \mathrm{g} / \mathrm{ml})$ dissolved in PBS containing 1\% BSA.

Ethyleneglycol-bis-succinimidyl-succinate (EGS) (Applichem, cat. A7846) fixation was performed as follows: growth medium was removed from cells, cells were first incubated for $1 \mathrm{~min}$ in BRB80 buffer containing 0.2\% IGEPAL-630 (80 mM K-PIPES, $\mathrm{pH} 6.8,1 \mathrm{mM} \mathrm{MgCl} 2$; $1 \mathrm{mM}$ EGTA, 0.2\% IGEPAL-630) then incubated for $10 \mathrm{~min}$ in BRB80 buffer containing $2 \mathrm{mM}$ EGS (freshly added from $250 \mathrm{mM}$ stock) and washed once with PBS. Subsequently, samples were stained with $2 \mu \mathrm{M}$ probes and Hoechst $33342(1 \mu \mathrm{g} / \mathrm{ml})$ dissolved in PBS containing 1\% BSA.

Living and fixed cells were imaged using fluorescence microscopy, and results are summarized (Supplementary Fig. 14 and Supplementary Table 2).

Toxicity test of SiR-tubulin and SiR-actin. Human primary dermal fibroblasts grown in $25-\mathrm{cm}^{2}$ dishes were detached using a trypsin/EDTA solution (Lonza, cat. BE17-161E), mixed with 
complete DMEM medium and seeded in glass-bottom 12-well or 24-well plates (total grown area, $48 \mathrm{~cm}^{2}$; MatTek, cat. P12GC1.0-14-F or P24G-1.5-13-F). Equal volume of complete DMEM medium containing Taxol, jasplakinolide or probes was added, and cells were grown in a humidified $5 \% \mathrm{CO}_{2}$ incubator at $37^{\circ} \mathrm{C}$ for $24 \mathrm{~h}$. Afterwards, equal volume of the complete DMEM medium containing $2 \mu \mathrm{g} / \mathrm{ml}$ Hoechst 33342 was added and incubated in a humidified $5 \% \mathrm{CO}_{2}$ incubator at $37{ }^{\circ} \mathrm{C}$ for $1 \mathrm{~h}$ before imaging. Cells were imaged, and results are summarized (Supplementary Fig. 8).

Cell preparation for SIM microscopy. Adult human dermal fibroblasts were cultured in DMEM supplemented with GlutaMAX-1 and 10\% FBS in a humidified $5 \% \mathrm{CO}_{2}$ incubator. Cells were seeded in glass-bottom $35-\mathrm{mm}$ dishes. Cells were stained with $\mathrm{SiR}$-tubulin and $\mathrm{SiR}$-actin probes at $2 \mu \mathrm{M}$ final concentration for $1-2 \mathrm{~h}$ at $37^{\circ} \mathrm{C}$ in complete DMEM. Depending on the experiment, cells were washed once with warm complete DMEM or imaged directly without washing. Cells were imaged, and results are summarized (Fig. 1c, Supplementary Fig. 10a-c and Supplementary Videos 6 and 7).

Cell preparation for STED microscopy. All the used cell lines were cultured in DMEM (Life Technologies, cat. 61965-059) supplemented with 10\% FCS (FCS, PAA, cat. A15-151) and pen/ strep (100 units/ml and $100 \mu \mathrm{g} / \mathrm{ml}$ respectively, BiochromAG, cat. A2213) at $37{ }^{\circ} \mathrm{C}$ and $5 \% \mathrm{CO}_{2}$. Cells were stained with SiRtubulin and SiR-actin probes at $2 \mu \mathrm{M}$ final concentration at $37{ }^{\circ} \mathrm{C}$ in HDMEM (phenol red-free DMEM-Invitrogen, cat. 31053-028-buffered with 10 mM HEPES) supplemented with $10 \%$ FCS and pen/strep. Imaging was performed in HDMEM buffer with $10 \%$ FCS.

Transfection of neurons with Lifeact was performed by means of a modified Semliki forest virus containing the pSCA3Lifeact-YFP (sequence available on http://figshare.com/articles/ pSCA3_Lifeact_YFP_txt/964797) construct with a CMV promoter. Imaging was performed on a previously described setup ${ }^{25}$. Axon initial segment was stained for 5 min at RT with anti-panneurofascin antibody (UC Davis/NIH NeuroMab Facility, clone A12/18) diluted 1:100 in artificial cerebrospinal fluid (ACSF buffer). After three washes, cells were incubated for $30 \mathrm{~s}$ with a goat anti-mouse Alexa 647 secondary antibody (Life Technologies, cat. A-21263), washed and imaged in ACSF buffer at RT.

For colocalization experiments with SiR-actin dye, neurons were incubated with $2 \mu \mathrm{M}$ of the probe for $1 \mathrm{~h}$ at $37^{\circ} \mathrm{C}$ in growth medium. Axon initial segment was stained as previously described. A goat anti-mouse Alexa Fluor 488 was used as secondary antibody (Life technologies, cat. A-11001).

Cells were imaged, and results are summarized (Fig. 2 and Supplementary Figs. 10d-1 and 11-13).

Wide-field microscopes. For imaging GFP, RFP and SiR, a Leica DMI6000B microscope equipped with a Leica HCX PL APO 100× 1.47-NA oil objective and a Hamamatsu-C9100 EM-CCD camera $(512 \times 512$ pixels $)$ was used. The following dichroic mirror and filters were used for GFP signal detection: excitation BP 470/40, emission BP 520/40 and dichroic mirror at $495 \mathrm{~nm}$. The following dichroic mirror and filters were used for RFP signal detection: excitation BP 530/35, emission BP 580/40 and dichroic mirror at
$565 \mathrm{~nm}$. The following dichroic mirror and filters were used for SiR signal detection: excitation BP 635/30, emission BP 700/72 and dichroic mirror at $650 \mathrm{~nm}$. $z$ stacks with voxel size of $240 \times$ $240 \times 692 \mathrm{~nm}^{3}$ were acquired, and images were presented as maximum-intensity projections (MIP) or the best focus plane.

For imaging Hoechst and SiR, a Zeiss Axiovert 200 inverted microscope equipped with an Olympus UPlanSApo 60×/1.2-NA W or Zeiss A-Plan $10 \times / 0.25$-NA Ph1 objectives and an AxioCam $(1,388 \times 1,040$ pixels $)$ was used. The following dichroic mirror and filters were used for Hoechst signal detection: excitation BP 365/12, emission LP 397 and dichroic mirror at $395 \mathrm{~nm}$. The following dichroic mirror and filters were used for SiR signal detection: excitation BP 640/30, emission BP 690/50 and dichroic mirror at $660 \mathrm{~nm}$.

Automated wide-field fluorescence microscopy was performed on a Molecular Devices ImageXpressMicro XL screening microscope equipped with reflection-based laser autofocus and a $10 \times / 0.5$-NA S Fluor dry objective (Nikon), controlled by in-house-developed MetaMorph macros ${ }^{26}$. Cells were maintained in a microscope stage incubator at $37^{\circ} \mathrm{C}$ in humidified atmosphere of $5 \% \mathrm{CO}_{2}$.

Confocal microscopes. Labeled samples were imaged on a Leica SP5 (Leica) upverted confocal microscope equipped with an HCX PL APO CS 63.0×/1.40-NA oil UV objective. Images were taken using an $8,000-\mathrm{Hz}$ resonant scanner, a voxel size of $40 \times 40 \times$ $126 \mathrm{~nm}^{3}$, a pinhole of $47.8 \mu \mathrm{m}(0.5 \mathrm{AU})$ and frame averaging of 3-12. The excitation light source was a white light laser set to the following values: $490 \mathrm{~nm}$ (power at 5-7\%, Bodipy FL channel) and $640 \mathrm{~nm}$ (power at 20\%, SiR channel). Emission light was registered with Leica $\mathrm{HyD}$ detectors set to the following spectral ranges: 500-550 nm (Bodipy FL channel) and 650-700 nm (SiR channel) and time-gating switched "on." Subsequently, deconvolution using a computed PSF was applied, and the colocalization of fluorescence signals was measured (Colocalization analyzer, Huygens Essentials package).

Confocal microscopy of HeLa cells shown in Supplementary Figure 3 was performed on a customized Zeiss LSM780 microscope using a $40 \times / 1.4$-NA oil DIC Plan-Apochromat objective (Zeiss), controlled by ZEN 2011 software and an autofocus macro (AutofocusScreen) kindly provided by J. Ellenberg. The microscope was equipped with an EMBL incubation chamber (European Molecular Biology Laboratory), providing a humidified atmosphere at $37^{\circ} \mathrm{C}$ with $5 \% \mathrm{CO}_{2}$.

SIM microscope. The images were acquired with a commercially available setup Nikon Eclipse Ti microscope equipped with a Nikon Apo TIRF 100×/1.49-NA objective and 2.5× internal magnification. 640-nm laser was used for excitation in combination with Cy5 HC filter set (AHF analysentechnik, cat. F36-523). The signal was detected by an electron-multiplying charge-coupled device (EMCCD) camera Andor DU897 (image size $512 \times 512$ pixels). Structured illumination is produced by projecting the image of a grating block onto the image plane inducing interferences with the sample image. The SIM images are reconstructed from the raw images through the Nikon proprietary software NISElement. The field of view is $32 \mu \mathrm{m} \times 32 \mu \mathrm{m}$ with $64 \mathrm{~nm} /$ pixel and $32 \mathrm{~nm} /$ pixel after reconstruction of SIM images. Two SIM modes can be chosen: 2D-SIM (does not allow optical sectioning) mode 
and 3D-SIM mode (allows optical sectioning). 2D-SIM: SIM data contains nine images, three phases (three grid shifts) and three grid angles. 3D-SIM: SIM data contains 15 images, 5 phases and 3 grid angles.

STED microscopes. The images were taken on a STED microscope described previously ${ }^{27}$. Fluorophores were excited with a pulsed laser diode at 640-nm wavelength (PicoQuant). To increase the resolution beyond the diffraction limit, we manipulated the beam of a pulsed, 775-nm-wavelength fiber laser (IPG Photonics) so that the focal intensity distribution exhibits a central intensity equal to 0 . The fluorescence outside the center of the focus is quenched by the 775-nm laser through stimulated emission, therefore leading to a subdiffraction-sized probing area. Using pulse energies of $3 \mathrm{~nJ}$, we achieved a resolution down to $<40 \mathrm{~nm}$. The images were acquired by scanning the sample with a piezo stage (Mad City Labs) and detecting the fluorescence with an avalanche photodiode (Micro Photon Devices). The SiR signal was acquired using a 660 - to $720-\mathrm{nm}$ detection window. A confocal (i.e., diffraction-limited resolution) channel with 470-nm excitation and 500- to 550-nm detection wavelengths was implemented to additionally image GFP. The overlay of the STED and confocal channels was adjusted and verified on 100-nm TetraSpeck fluorescent beads (Life Technologies).

An alternative STED microscope was equipped with an excitation 635-nm pulsed diode laser and driver (LDH-D-C-635 and PDL 800-B, PicoQuant, 100-ps pulse width) and STED titanium-sapphire laser, mode locked at $770 \mathrm{~nm}$ for STED (Mira900, Coherent). The repetition rate of the STED pulse is $76 \mathrm{MHz}$, and the pulse length is stretched from 150 fs to about 200 ps by $121-\mathrm{m}$ fiber (PMJ-A3HPC, AMS Technologies). Excitation is synchronized to the STED laser and triggered at every seventh pulse to reduce the repetition rate to approximately $10 \mathrm{MHz}$. Detector: APDs (SPCMAQR-13-FC, PerkinElmer Optoelectronics). The focal STED doughnut was created by passing the STED beam to a polymer vortex phase plate (RPC Photonics) and fed into a 1.4-NA objective lens (HCX PL APO $100 \times / 1.40$ - to 0.70-NA, Leica).

Lifeact-YFP experiments were performed on the setup described in the paper Urban et al. ${ }^{25}$. Briefly, a pulsed-laser diode (Toptica Photonics) was used to deliver excitation pulses with a 490-nm wavelength, 100-ps duration and average focal power of $0.7-2.8 \mu \mathrm{W}$. The pulses for the STED beam were delivered by a Ti:sapphire laser (MaiTai; Spectra-Physics) operating at $80 \mathrm{MHz}$ and emitting at $\lambda=795 \mathrm{~nm}$; the pulses were converted to $\lambda=595 \mathrm{~nm}$ by an optical parametric oscillator (APE).

Processing and visualization of acquired images. All acquired or reconstructed images were processed and visualized using Fiji ${ }^{28}$. For visualization of super-resolution microscopy images, the "Red hot" lookup table was used. Deconvolution of STED images of the centrosomes was performed using the Richardson-Lucy algo rithm and ImSpector (Max-Planck Innovation) software. Acquired SIM images were reconstructed with Nikon Nis-Elements 4.11. Line profiles were measured using the "Straight line" tool with line width set to 3 pixels. All profiles were fitted to Gaussian of Lorentz distributions using appropriate tools available on OriginPro 9 (http://www.originlab.com/).

Microscopy images shown in Supplementary Figures 3 and 9 and in Supplementary Videos $\mathbf{1 - 5}$ were linearly contrast adjusted using Fiji. Proliferation and mitotic duration was measured using the CellCognition software (http://www.cellcognition.org/) by automated cell detection, tracking over time, and morphology classification by supervised machine learning as described in ref. 26. In brief, cells were segmented by local adaptive thresholding on the chromatin marker. Single cells were tracked over time on the basis of nearest-neighbor calculations that permit cell object splits and merges. The classification of cells into interphase, prometaphase, metaphase, anaphase and dead cells was by supervised machine learning using support vector machines on the basis of texture and contour features. Mitotic events were detected in full-length trajectories on the basis of interphase-prometaphase transitions. Mitotic duration was calculated from prometaphase onset until anaphase onset. Proliferation index was calculated by dividing all live cells from the last movie frame $(23 \mathrm{~h})$ by all live cells from the first movie frame.

Cleavage-furrow ingression efficiency was quantified by visual inspection of the full-length movies $(0.5-23.5 \mathrm{~h}$ after drug addition; data shown in Supplementary Fig. 9 and Supplementary Videos 1-5). Cells entering anaphase were identified on the basis of the chromatin marker H2B-mRFP, and furrow ingression was then scored on the basis of the plasma-membrane marker MyrPalm-mEGFP. Multinucleation was scored by visual inspection of the last movie frame $23 \mathrm{~h}$ after drug addition on the basis of two or more separate nuclei marked by H2B-mRFP within one cell boundary visualized by MyrPalm-mEGFP. The multinucleation index was calculated by dividing the number of multinucleated cells by the total number of live interphase cells.

General statistical analysis. All measured parameters were measured at least in duplicate and presented as mean with s.d. or standard error range as well as sample size indicated. In vitro replicates are measured on different days using the same setup. Each sample was measured at least in technical duplicate. Cellular imaging experiments were performed at least twice on different days. Images at several different places of cell culture were acquired. If applicable, these parameters were indicated in the figure legends of the manuscript or supplementary information. Cell staining with probes was independently reproduced by the three groups of D.W.G., S.W.H. and K.J.

20. Bonne, D., Heuséle, C., Simon, C. \& Pantaloni, D. J. Biol. Chem. 260, 2819-2825 (1985).

21. Cooper, J.A., Walker, S.B. \& Pollard, T.D. J. Muscle Res. Cell Motil. 4, 253-262 (1983).

22. Viswanadhan, V.N., Ghose, A.K., Revankar, G.R. \& Robins, R.K. J. Chem. Inf. Comput. Sci. 29, 163-172 (1989).

23. Byers, T.J. \& Branton, D. Proc. Natl. Acad. Sci. USA 82, 6153-6157 (1985).

24. Behnke, 0. J. Ultrastruct. Res. 31, 61-75 (1970).

25. Urban, N.T., Willig, K.I., Hell, S.W. \& Nägerl, U.V. Biophys. J. 101, 1277-1284 (2011).

26. Held, M. et al. Nat. Methods 7, 747-754 (2010).

27. Göttfert, F. et al. Biophys. J. 105, L01-L03 (2013).

28. Schindelin, J. et al. Nat. Methods 9, 676-682 (2012). 